\title{
Asteroseismology of the SPB star HD 21071
}

\author{
Wojciech Szewczuk and Jadwiga Daszyńska-Daszkiewicz
}

\author{
Instytut Astronomiczny, Uniwersytet Wrocławski, Wrocław, Poland
}

email: szewczuk@astro.uni.wroc.pl

\begin{abstract}
We perform mode identification for the frequency peaks detected in the light variation of HD 21071 including the effects of rotation via the traditional approximation. We find the angular numbers $(\ell, \mathrm{m})$ for all observed frequencies and limit the range of the rotational velocity. In the next step, we make an attempt towards seismic modelling of the star in order to constrain its global parameters.
\end{abstract}

Keywords. stars: oscillations, early-type, rotation

\section{Introduction}

HD 21071 (V576 Per) is a slowly pulsating B-type star with the brightness of $V=6.08$ mag, the spectral type of B5IV or B7V (Reed 2005), rotating at a speed of at least $50 \mathrm{~km} / \mathrm{s}$ (Abt et al. 2002). The effective temperature $T_{\text {eff }}=4.164(7)$ and metallicity $Z=0.0082(16)$ was derived from the IUE ultraviolet spectra by Niemczura (2003). The luminosity of $\log L / L_{\odot}=2.444(76)$, needed to put the star on the H-R diagram, was determined in this paper. Using the Geneva time-series photometry, De Cat et al. (2007) found four pulsational frequencies: $\nu_{1}=1.18843, \nu_{2}=1.14934, \nu_{3}=1.41968$ and $\nu_{4}=0.95706 \mathrm{c} / \mathrm{d}$. The dominant one $\nu_{1}$ was also found in the spectroscopic data (De Cat 2002).

\section{Mode identification and frequency fitting}

In order to make mode identification we apply the method proposed by DaszyńskaDaszkiewicz et al. (2008), which takes into account the Coriolis force in the framework of the traditional approximation. The results are presented in Tab. 1. We considered the models from the centre and four edges of the error box. For $\nu_{4}$ in the model \#4 we were unable to find mode identification in the common range of $V_{\text {rot }}$ with other frequencies. They are indicated by a question mark. The similar situation take place for the scenario $\nu_{4}(\ell=1, m=0)$ in the model \#5. Depending on the model parameters, the rotation velocity of HD21071 ranges from about 150 to $250 \mathrm{~km} \mathrm{~s}^{-1}$.

\section{Frequency fitting}

Fitting the two $\left(\nu_{1}, \nu_{2}\right)$ and three $\left(\nu_{1}, \nu_{2}, \nu_{3}\right)$ frequencies was performed for models with $\log L / L_{\odot}$ and $\log T_{\text {eff }}$ from the $3 \sigma$ error box. We considered the rotational velocities, $V_{\text {rot }}$, in the range from 150 to $255 \mathrm{~km} \mathrm{~s}^{-1}$. In Fig. 1 the results are shown for $V_{\text {rot }}=160 \mathrm{~km} \mathrm{~s}^{-1}$. There are six combinations of the radial orders that fit two frequencies and only one $\left(g_{13}\right.$, $\left.g_{14}, g_{9}\right)$ that fits three frequencies for the depicted value of $V_{\text {rot }}$. 
Table 1. Mode identification for models from the centre and edges of the error box.

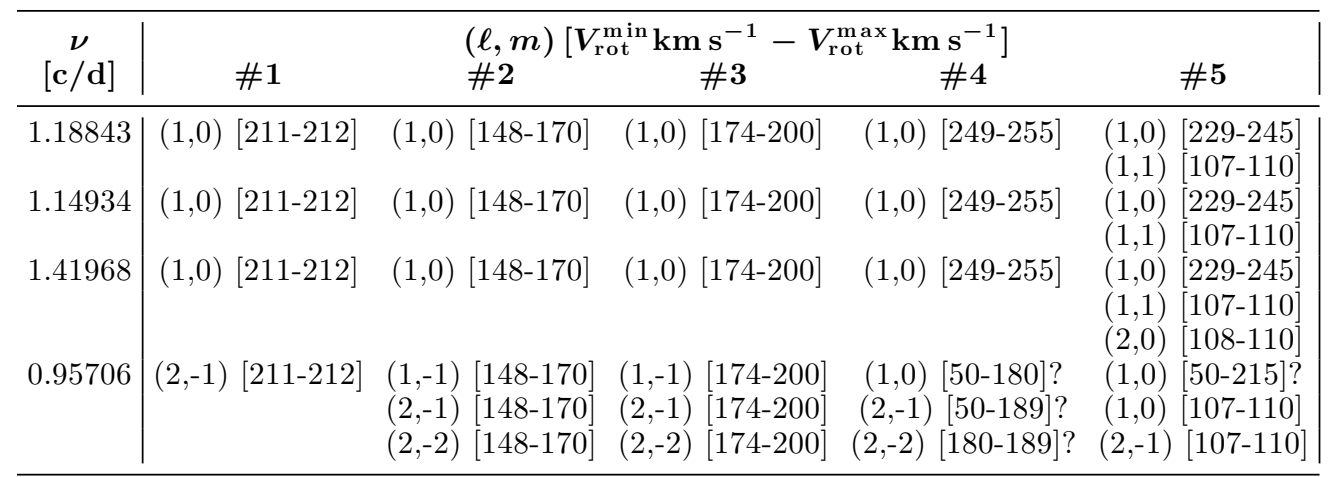

Notes: \#1: $\log T_{\text {eff }}=4.164, \log L / L_{\odot}=2.444 ; \# \mathbf{2}: \log T_{\text {eff }}=4.171, \log L / L_{\odot}=2.368 ; \# \mathbf{3 :} \log T_{\text {eff }}=4.157$, $\log L / L_{\odot}=2.368 ; \# 4: \log T_{\text {eff }}=4.171, \log L / L_{\odot}=2.520 ; \# \mathbf{5 :} \log T_{\text {eff }}=4.157, \log L / L_{\odot}=2.520$

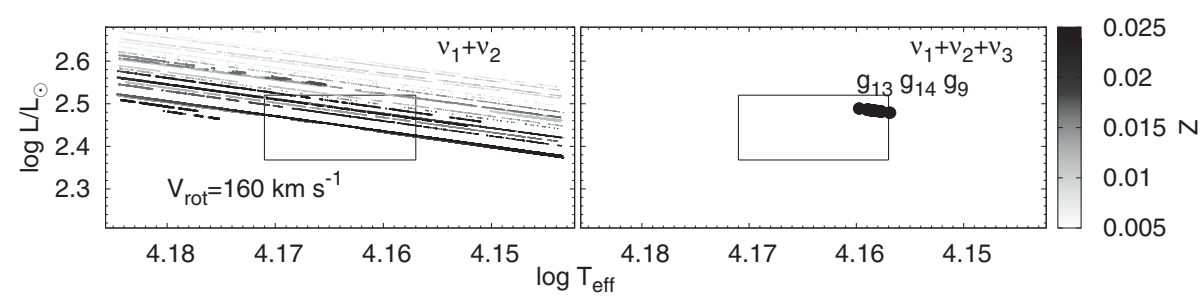

Figure 1. The seismic models of HD 21071 on the H-R diagram for $V_{\text {rot }}=160 \mathrm{~km} \mathrm{~s}^{-1}$. Only models with unstable modes are shown. Grayscale codes metallicity $Z$. The left panel: the seismic models fitting $\nu_{1}$ and $\nu_{2}$. The right panel: the seismic models fitting $\nu_{1}, \nu_{2}$ and $\nu_{3}$.

\section{Conclusions}

Mode identification in the framework of the traditional approximation is slightly depended on the model. Despite of this fact, we succeeded in determining the angular numbers $(\ell, m)$ of the frequencies observed in HD 21071. The simultaneously determined value of the rotational velocity is more sensitive to the adopted stellar parameters and we could constrain its value to the range of about $(150-250) \mathrm{km} \mathrm{s}^{-1}$.

In the next step, we try to find rotating models which reproduce the well identified frequencies. There are models which fit two and three observed frequencies in the determined range of $V_{\text {rot }} \in(150-250) \mathrm{km} \mathrm{s}^{-1}$. When only unstable modes are considered, the seismic models fitting $\nu_{1}$ and $\nu_{2}$ become more luminous and their effective temperatures increase with the rotation rate. Above $V_{\text {rot }}=180 \mathrm{~km} / \mathrm{s}$ there are no models with unstable modes fitting the three frequencies.

\section{Acknowledgements}

WS was financially supported by the Polish NCN grant DEC-2012/05/N/ST9/03905 and JDD by the Polish NCN grants 2011/01/M/ST9/05914, 2011/01/B/ST9/05448.

\section{References}

Abt, H. A., Levato, H., \& Grosso, M. 2002, ApJ 573, 359

Daszyńska-Daszkiewicz, J., Dziembowski, W. A., \& Pamyatnykh, A. A. 2008, Journal of Physics Conference Series 118(1), 012024 
De Cat, P. 2002, in IAU Colloq. 185: Radial and Nonradial Pulsationsn as Probes of Stellar Physics, Vol. 259 of Astronomical Society of the Pacific Conference Series, p. 196

De Cat, P., Briquet, M., Aerts, C., et al. 2007, A\&A 463, 243

Niemczura, E. 2003, A\&A 404, 689

Reed, B. C. 2005, AJ 130, 1652

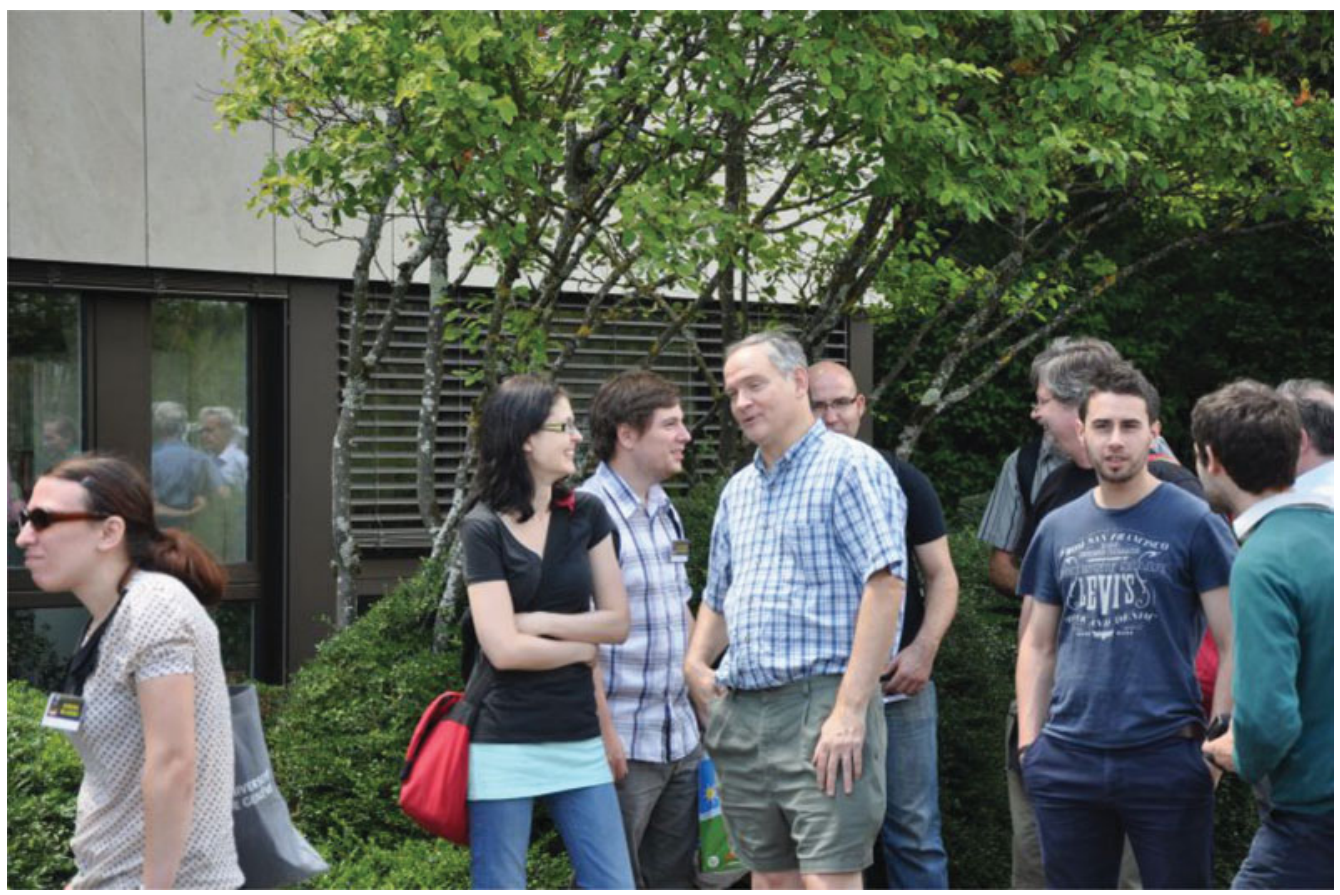

Participants during the CERN visit 